\title{
Ejercicio en Ratas con Osteoartritis. Aspectos Morfológicos y una Revisión de la Literatura
}

\author{
Exercise in Rats Osteoarthritis. Morphological Aspects and Literature Review
}

\author{
Claudia Andrea Vargas ${ }^{1,2}$; Karina A. Sandoval ${ }^{2}$; C. A. Salvo ${ }^{3}$; Mariano del Sol ${ }^{1,5}$ \& Nicolás Ernesto Ottone ${ }^{1,4,5}$
}

VARGAS, C. A.; SANDOVAL, K. A.; SALVO, C. A.; DEL SOL, M. \& OTTONE, N. E. Ejercicio en ratas con osteoartritis. Aspectos morfológicos y una revisión de la literatura. Int. J. Morphol., 38(2):481-491, 2020.

RESUMEN: La osteoartritis (OA) es una enfermedad crónica, degenerativa, muy invalidante, que destruye en forma gradual y progresiva el cartílago articular en diversas regiones: rodillas, caderas, hombros, manos, tobillos y columna vertebral. En este sentido, el ejercicio ha sido descrito como la intervención no farmacológica más recomendada para pacientes con OA. La práctica regular de ejercicio es considerada un componente integral del estilo de vida saludable; sin embargo, su efecto en el cartílago se mantiene como objeto de debate y especulaciones, así como la relación del ejercicio con el desarrollo de OA. Algunos estudios de modelos animales sugieren que el ejercicio puede ser beneficioso para la salud del cartílago, mientras otros demuestran su efecto nocivo. Una explicación general a estos resultados inconsistentes es que el correr a intensidad moderada tiene efectos beneficiosos, mientras que correr "vigorosamente" o de manera "extenuante" lleva a un efecto nocivo. El objetivo de este trabajo consistió en realizar una revisión de la literatura acerca de los efectos del ejercicio sobre el cartílago artícular, especialmente enfocado a modelos animales experimentales con ratas.

PALABRAS CLAVE: Osteoartritis; Ejercicio; Ratas; Morfología.

\section{INTRODUCCIÓN}

La osteoartritis (OA) es la más común de las enfermedades reumáticas crónicas (Kuyinu et al., 2016). Se manifiesta por dolor, deformidad e incapacidad funcional principalmente de las articulaciones con gran movilidad o que soportan peso. Debido al progresivo envejecimiento de la población mundial y la mayor prevalencia de enfermedades crónico-degenerativas, la osteoartritis se ha convertido en un problema de salud pública que debe ser reconocido en forma temprana. El $15 \%$ de la población mundial por encima de los 60 años padece OA y además es considerada diez a doce veces más frecuente que la artritis reumatoide (Wieland et al., 2005). No existe una causa única para el desarrollo de la OA, ya que se trata de una enfermedad multifactorial entre cuyos factores predominan la edad, obesidad, lesiones, género y predisposición genética, siendo característica primordial de todos los casos la degradación progresiva del cartílago articular (Pottie et al., 2006; Lavalle Montalvo, 2010). Las ratas son el modelo ampliamente utilizado en investigaciones experimentales y testeo de drogas tóxicas (Bendele, 2001). El modelo animal en ratas puede ser utilizado para entender los procesos de lesiones de cartílago y para desarrollar y evaluar modalidades de tratamiento para prevenir el daño del cartílago. Demuestran además que es factible para evaluaciones histopatológicas de la degeneración de cartílago como pérdida del mismo, degeneración de los meniscos, y el patrón de edema de la médula ósea después de un desgarro masivo de los tendones de los músculos rotadores de la articulación humeral (Link \& Li, 2011).

La práctica regular de ejercicio es considerada un componente integral del estilo de vida saludable; sin embargo, su efecto en el cartílago se mantiene como objeto de debate y especulaciones, así como la relación del ejercicio con el desarrollo de OA (Hansen et al., 2012). El correr es una de las actividades más comunes que soportan peso y su efecto en el cartílago de la articulación de la rodilla es conflictivo. Algunos estudios de modelos animales sugieren que

\footnotetext{
${ }^{1}$ Doctoral Program in Morphological Sciences, Faculty of Medicine, Universidad de La Frontera, Temuco, Chile.

${ }^{2}$ Department of Physical Education, Sports and Recreation, Faculty of Education, Universidad de La Frontera, Temuco, Chile.

${ }^{3}$ Undergraduate Student of Physical Education, Sports and Recreation, Faculty of Education, Universidad de La Frontera, Temuco, Chile.

${ }^{4}$ Laboratory of Plastination \& Anatomical Techniques, CICO Research Centre of Dental Sciences, Adults Integral Dentistry Department, Dental School, Universidad de La Frontera, Temuco, Chile.

${ }^{5}$ Center of Excellence in Morphological and Surgical Studies (CEMyQ), Faculty of Medicine, Universidad de La Frontera, Temuco, Chile.
} 
el ejercicio puede ser beneficioso para la salud del cartílago (Kiviranta et al., 1988; Otterness et al., 1998), mientras otros demuestran su efecto nocivo (Ni et al., 2011; Lee et al., 2011). Una explicación general a estos resultados inconsistentes es que el correr a intensidad moderada tiene efectos beneficiosos, mientras que correr "vigorosamente" o de manera "extenuante" lleva a un efecto nocivo. El correr de manera "extenuante" es considerado como un modelo fiable de OA en animales (Lee et al.). Una manera común de normalizar la intensidad del ejercicio es expresarlo como porcentaje de consumo máximo de oxígeno (VO2max) el cual es generalmente considerado el mejor indicador de la resistencia cardiorespiratoria y de la capacidad aeróbica (Åstrand \& Rodahl, 1986). La evidencia ha demostrado que a mayor intensidad del ejercicio, mayor es el incremento de la capacidad aeróbica (Wenger \& Bell, 1986). Bedford et al. (1979) demostraron el porcentaje respectivo de VO2max relacionado a varios programas de entrenamiento en corredora para ratas Wistar, baja intensidad ( $60 \%$ VO2max $)$, media ( $75 \%$ VO2max) y alta intensidad ( $90 \%$ VO2max). La relación entre la intensidad al correr y la carga inducida al cartílago debería serclarificada en futuros estudios. "La dosis" óptima de ejercicio no es precisa (Ni et al., 2013). Estudios demuestran que el estrés mecánico excesivo puede dañar directamente a la matriz extracelular del cartílago, cambiar el balance en los condrocitos para favorecer la actividad catabólica sobre la anabólica (Sun, 2010). Otros estudios moleculares han revelado que el estímulo específico biomecánico e interacciones celulares, generan señales intracelulares que son poderosos supresores o inductores de genes pro-inflamatorios en los condrocitos (Knobloch et al., 2008). Murray et al. (2001) encontraron que el efecto de la carrera de alta intensidad, comparada con la de baja intensidad, estaba asociada con el aumento del grosor del hueso subcondral, aumento de remodelación ósea y reducción de reabsorción ósea en equinos.

Los efectos del ejercicio sobre el cartílago articular son un tema controversial, donde la dosis del ejercicio puede ser considerada clave para determinar la regeneración o deterioro del cartílago articular, por lo que el objetivo de este estudio, fue realizar una revisión de los artículos científicos experimentales en ratas, para dilucidar los efectos del ejercicio sobre el cartílago articular.

\section{MATERIAL Y MÉTODO}

Se realizó una revisión sobre ejercicio y osteoartritis en ratas, la búsqueda se realizó en las bases de datos "Embase", "Scopus", "Medline Complete", "Pubmed" y "Rehabilitation and Sport", las palabras claves utilizadas fueron: "Osteoarthritis," "Exercise", "Rats". Esta búsqueda se realizó entre el 18 de abril y el 6 de junio de 2019, encontrando 1092 artículos en total. Los términos fueron buscados como palabras claves, en el título y/o resumen y texto. Al comienzo tres observadores, (KS; CS Y CV), buscaron

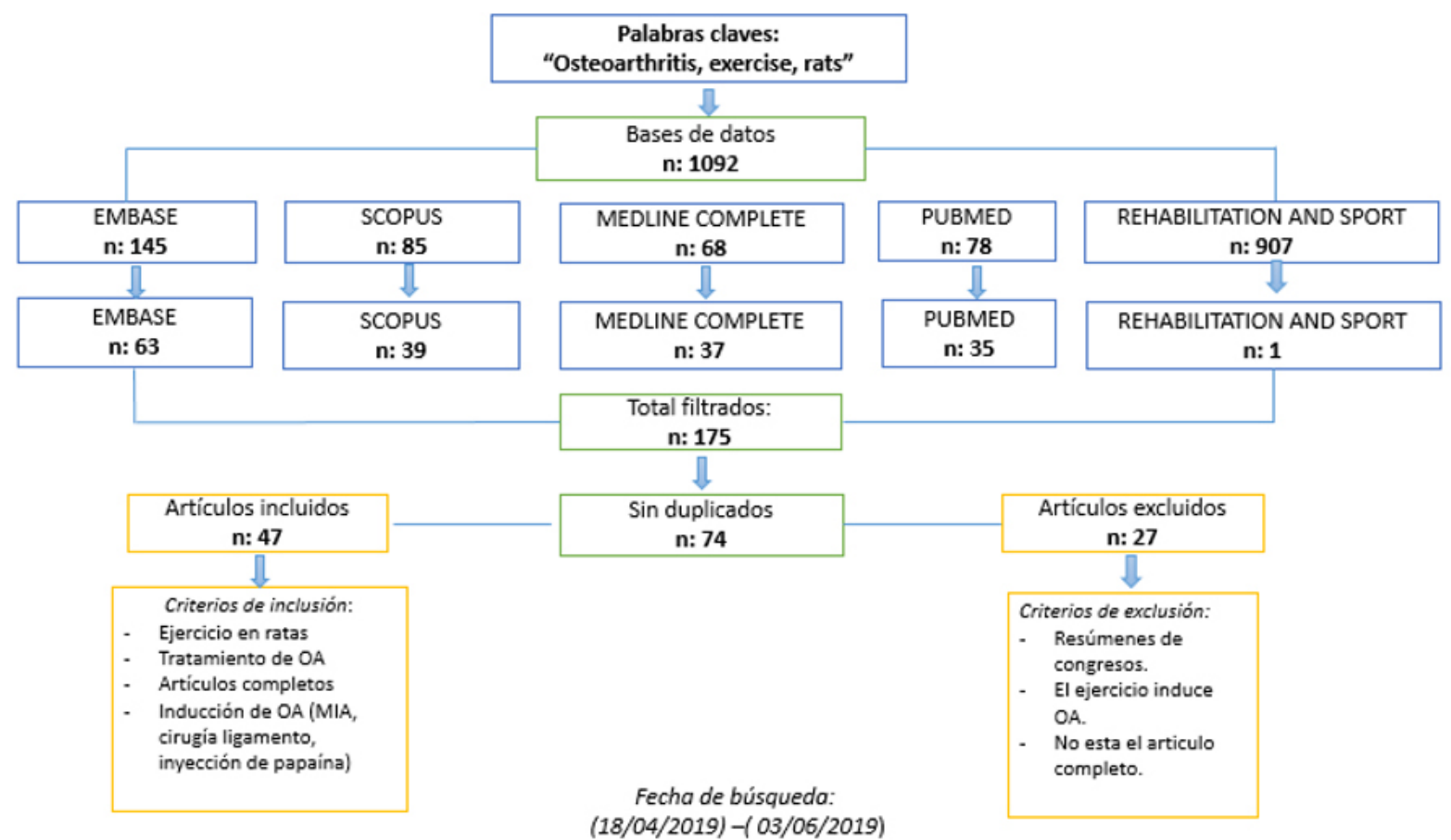

Fig. 1. Diagrama de flujo selección de artículos. 
las palabras claves en los títulos, resúmenes y textos. Posteriormente, filtraron los artículos de cada base de datos por el criterio de inclusión: Ejercicio en ratas con Osteoartritis. Fueron incluidos 174 artículos en total en todas las bases de datos. Luego se filtraron los duplicados, quedando 74 artículos no duplicados (Fig.1). De estos artículos, se incluyeron los que cumplían con los siguientes criterios: Texto completo, Tratamiento de OA con ejercicio, OA inducida (por MIA, cirugía, papaína), seleccionando un total de 47 artículos que cumplían con los criterios planteados.

De acuerdo a la revisión realizada de diversos estudios experimentales, se encontraron artículos que demostraban resultados de efectos positivos y negativos del ejercicio sobre el cartílago articular, dependiendo generalmente de los protocolos de entrenamiento que se llevaban a cabo y de las variables involucradas.

\section{RESULTADOS Y DISCUSIÓN}

Dentro de los artículos que mostraron efectos positivos del ejercicio, encontramos a Assis et al. (2015) los cuales evaluaron los efectos del ejercicio aeróbico y terapia de láser sobre las modificaciones degenerativas y los mediadores inflamatorios en el cartílago articular de rodilla de rata, con OA inducida por cirugía, (cortando el ligamento cruzado anterior), concluyendo que los grupos tratados con ejercicio y láser por separado y combinados, mostraron un mejor patrón organizacional del tejido, con menos fibrilación e irregularidades a lo largo de la superficie articular y organización de condrocitos, un menor proceso degenerativo medido por la escala OARSI y valores más altos de grosor articular.

Zhang et al. (2019) estudiaron las alteraciones del cartílago articular en rodilla de rata con OA inducida por MIA (monoiodo acetato de sodio), luego de la aplicación de un protocolo de ejercicio moderado en corredora, concluyendo que el ejercicio en corredora por 30 minutos al día, por 5 días a la semana, durante 4 semanas, tuvo un evidente factor protector en el cartílago articular y que podría promover la autofagia en todas las zonas del cartílago articular. La autofagia es un mecanismo de homeostasis celular esencial en células Eucariotas, involucra biosíntesis continua y renovación de los organelos celulares y macromoléculas. Estudios previos, han reportado que la autofagia en condrocitos, principalmente, sirve como respuesta protectora de las células a una estimulación negativa como la hipoxia (Milner et al., 2006), podría además ser un proceso esencial para la diferenciación y maduración de los condrocitos, lo que ocurre constantemente durante el proceso patológico de la OA (Srinivas et al., 2009). En conclusión, el estudio de Zhang et al. mostraron una potente evidencia sobre la hipótesis de que el ejercicio moderado en corredora, tiene un efecto protector en el cartílago articular de ratas con OA inducida por inyección intraarticular de MIA. Treinta minutos de ejercicio en corredora tuvo un evidente efecto protector en el cartílago articular de ratas con OA inducida por MIA y podría promover la autofagia en el cartílago articular.

Cormier et al. (2017) realizaron experimentos para ver los efectos de la marcha sobre rueda en la asimetría de la distribución del peso en los miembros posteriores, provocada por la OA y la micro arquitectura del hueso trabecular. Para la ejecución de los experimentos se utilizaron ratas adultas que realizaron un protocolo de ejercicio voluntario que duro 7 a 21 días sobre rueda rodante, luego se les indujo OA de rodilla con inyección de monoiodo acetato de sodio (MIA). Como resultado se determinó que 7 de días de ejercicio previo no altera la asimetría de peso provocada por MIA, sin embargo con 21 días de entrenamiento, se observó una reversión completa de la asimetría de peso. Se concluyó que el ejercicio voluntario ayuda a proteger contra el dolor de la OA y los efectos son variables de acuerdo a la duración del período de intervención con ejercicio, esto se asocia con la modificación del hueso trabecular.

Cifuentes et al. (2010) estudiaron los efectos del ejercicio en corredora sobre el estrés oxidativo del cartílago de rodilla de rata con OA inducida por MIA, donde los datos histológicos indicaron que los animales sometidos al ejercicio en corredora, mostraron una tasa de preservación de contenido de proteoglicanos en la zona superficial e intermedia del cartílago. Demostrando además que el ejercicio aumenta el mecanismo de defensa contra el estrés oxidativo. El tejido articular es considerado un tejido hipoxico, debido a que es avascular. El rol de cambio de los niveles de oxígeno durante el proceso de degradación es de gran interés. Una producción elevada de especies reactivas de oxígeno (ROS) y disminución de antioxidantes han sido observadas en condiciones patológicas articulares de inflamación. Se concluye que altos niveles de ejercicio, aumentan el riesgo de padecer OA, pero niveles moderados son beneficiosos para la articulación.

Qian et al. (2014) evaluaron los efectos del movimiento pasivo y moderado sobre OA de rodilla, provocada por fractura, logrando OA leve y moderada. Los grupos experimentales fueron ejercitados en corredora, durante tres y seis semanas por una hora al día a una velocidad de $15 \mathrm{~m} /$ min. Evaluaron los efectos del movimiento sobre el grosor del cartílago, escala de Mankin, el colágeno de la matriz 
del cartílago, contenido de proteoglicanos y la estructura morfológica del cartílago en ratas con OA, que fueron medidas en diferentes estadios degenerativos causados por fractura de rodilla. El contenido de proteoglicanos en la matriz del cartílago, fibras de colágeno tipo II y el número de células bajo apoptosis fueron medidas semicuantitativamente. Concluyeron que un movimiento pasivo adecuado, puede reparar y mejorar el metabolismo de los condrocitos y retrasar el progreso degenerativo del cartílago articular con OA provocado por fractura de rodilla. Sin embargo, para estadíos medios de OA, el movimiento pasivo no exhibió un efecto reparativo significativo en el cartílago articular. Esta terapia aumenta los niveles de proteoglicanos y fibras colágenas reduciendo su descomposición, de éste modo, mejorando la fuerza del ligamento articular y la estabilidad de la articulación.

Ijama et al. (2015) evaluaron los efectos de la caminata suave en corredora para ratas, sobre los cambios del hueso subcondral y el cartílago, en un modelo experimental de desestabilización de menisco medial. Utilizaron ratas Wistar y fueron divididos en dos grupos: sedentario y con caminata en corredora. El ejercicio comenzó dos días post cirugía, que incluía caminata a una velocidad de $12 \mathrm{~m} / \mathrm{min}$, $30 \mathrm{~min} /$ día, 5 días/semana, durante 4 semanas. Los cambios del hueso subcondral y cartílago fueron evaluados por micro CT, análisis histológicos y biomecánicos. Las ratas con caminata mostraron, en el examen de micro CT, una tendencia a suprimir el crecimiento del hueso subcondral, además de mostrar una actividad de osteoclastos por tinción positiva de ácido fosfatasa tartrato-resistente 4 semanas post cirugía. Estos resultados coinciden con la prevención de la degradación del cartílago medida por escala OARSI y análisis biomecánico. Además la caminata en corredora podría haber suprimido el aumento de muerte de osteocitos. Estos resultados indican que existe un link biomecánico y biológico entre el cartílago y el hueso subcondral. Los efectos preventivos de la caminata en corredora sobre el deterioro del hueso subcondral, podrían ser parcialmente explicados por los efectos condroprotectores.

Blazek et al. (2016) desarrollaron un estudio donde se busca demostrar la importancia que tiene el ejercicio para conservar la integridad del cartílago en articulaciones sanas. Se utilizaron ratas Sprague-Dawley hembras sanas que fueron sometidas a ejercicios de baja intensidad durante 2 , 5 y 15 días. Luego se realizó un análisis de microarrays para determinar la expresión genética y la interpretación biológica de las redes moleculares. En la conclusión se destaca que el ejercicio es un regulador transcripcional de varias vías metabólicas del cartílago sano, atenúa vías inflamatorias y fortalece el cartílago. Además facilita la profilaxis de enfermedades artríticas en sujetos sanos.
Siebelt et al. (2014a) investigaron el uso preventivo del alendronato (ALN) en la reabsorción ósea subcondral osteoclástica relacionada con la $\mathrm{OA}$ en ratas. Para este estudio se indujo OA severa en rodilla con inyecciones de papaína combinado con un ejercicio moderado durante 6 semanas, recorrían $500 \mathrm{~m}$ en cinta rodante al día 5 veces a la semana. Durante el experimento la mitad del grupo recibió 3 veces a la semana inyecciones subcutáneas de ALN. Como resultado se halló una pérdida ósea subcondral y una formación de osteofitos reducida en las ratas que se inyectaron con ALN, además redujo la degradación del cartílago. Junto con el ejercicio moderado la producción de glicosaminoglicanos en el cartílago sano mostró un aumento. En conclusión el tratamiento con ALN combinado con ejercicio genera un efecto positivo en las articulaciones de control sana.

Ni et al. (2012) se plantearon como objetivo de estudio comprender los cambios en la expresión de lubricina del cartílago por inmunolocalización, en ratas sometidas a ejercicios de carrera de diferentes intensidades. Para esto, las ratas fueron divididas en 4 grupos; uno de control sedentario (CON), uno de carrera de baja intensidad (LIR), uno de intensidad moderada (MIR) y uno de carrera de alta intensidad (HIR). El protocolo de ejercicio fue aplicado durante 8 semanas y las cargas variaron de acuerdo al grupo. Los resultados del análisis de inmunohistoquímica para la lubricina, mostraron un contenido mayor de lubricina en el grupo MIR que el grupo CON y se encontró un contenido significativamente menor de lubricina en el grupo HIR, en donde se observaron cambios osteoartríticos. En conclusión hay un marcado efecto en la intensidad de ejecución del ejercicio y en la expresión de lubricina.

Castrogiovanni et al. (2019) investigaron los efectos que tiene la actividad física moderada sobre a osteoartritis de rodilla, evaluando la expresión de biomarcadores de la OA (IL-1 $\beta$, IL-6, TNF- $\alpha$ y MMP-13) y los biomarcadores antiinflamatorios y condroprotectores de sinoviocitos tipo B (IL-4, IL-10, lubricina). Para provocar la OA se utilizó la cirugía de corte del ligamento cruzado anterior (CLCA). Las ratas se dividieron en cuatro grupos: control sanas (grupo 1), ratas que realizan ejercicio de intensidad moderada (EIM) (grupo2), ratas CLCA con OA (grupo3) y ratas CLCA con OA y sometidas EIM (grupo4). El protocolo de ejercicio que se llevó a cabo fue de 25 min 3 veces a la semana con ejercicio progresivo de intensidad leve a moderada, durante 12 semanas. Los análisis fueron realizados con tinción de Hematoxilina Eosina, histomorfometría e inmunohistoquímica. En el grupo 3, se detectó que los biomarcadores de OA (IL-1 $\beta$, IL-6, TNF- $\alpha$ y MMP-13), tuvieron un aumento significativo, y la IL-4, IL-10 y lubricina fue más baja que en los otros grupos experimentales. El grupo 4 (ratas CLCA con OA y sometidas EIM), 
mostró una expresión disminuida de los biomarcadores de OA y una expresión de biomarcadores condroprotectores aumentada, concluyendo que el ejercicio de intensidad moderada, tiene un efecto beneficioso sobre la membrana sinovial y en consecuencia, en la preservación del cartílago articular.

Assis et al. (2016) evaluaron los efectos de un protocolo de ejercicio y terapia láser de baja intensidad y la asociación de ambos tratamientos sobre la atrofia musculoesquelética utilizando un modelo experimental de osteoartritis de rodilla (OA). Los resultados mostraron que ambos grupos entrenados (irradiados o no) presentaron un aumento significativo en el área de la sección transversal muscular y una disminución en la densidad de la fibra muscular en comparación con el grupo control de OA de rodi1la. Éstos resultados sugieren que el entrenamiento con ejercicios y la terapia con láser de baja intensidad fueron eficaces para prevenir las alteraciones musculo-esqueléticas relacionadas con la atrofia causada por el proceso degenerativo inducido por la $\mathrm{OA}$ de rodilla.

Hong et al. (2014) evaluaron los efectos de la melatonina con dosis graduadas en la síntesis de la matriz extracelular y la muerte celular en respuesta al daño del cartílago, la intervención se realizó utilizando el tratamiento con melatonina (MT) y melatonina combinada con ejercicio en cinta rodante $(\mathrm{MT}+\mathrm{Ex})$, el resultado sugiere que la melatonina con ejercicio en cinta rodante puede tener efectos tanto preventivos como sinérgicos en el rescate de la degeneración del cartílago y es más efectiva en la fase inicial.

Na et al. (2014) investigaron la correlación entre el efecto del ejercicio en cinta rodante y el cambio en el nivel de proteínas séricas en ratas con OA, inducida por MIA. Utilizaron un espectrómetro de masas para realizar el análisis. Las ratas se dividieron aleatoriamente en cinco grupos: Grupo control, MIA sin ejercicio, MIA con ejercicio de baja intensidad, MIA con ejercicio de mediana intensidad y MIA con ejercicio de alta intensidad. Después de 4 semanas de entrenamiento en cinta rodante, analizaron el suero de cada rata encontrando que diecisiete proteínas estaban elevadas en las muestras del grupo de osteoartritis por inyección de MIA más de 1,5 veces en comparación con el grupo control. Los resultados demostraron que el componente complementario 9 (C9) estaba aumentado en el suero de las ratas con OA inducida por MIA, lo que fue validado por Western blot. La proteína C9 está relacionada con la inflamación y se vio disminuida después del ejercicio en cinta rodante, siendo más baja en el grupo de ejercicio moderado. Concluyendo que el ejercicio en corredora a una velocidad apropiada puede ser recomendado en pacientes con OA.
Nam et al. (2017) investigaron el efecto del cambio de velocidad de la caminata en el ángulo de la rodilla de ratas con OA, inducida por MIA. Las ratas fueron divididas en dos grupos: sin cambio de velocidad y con cambio de velocidad. La velocidad para el grupo control fue continua, y para el grupo con MIA, la velocidad fue variando. El protocolo de ejercicio fue por dos semanas, 4 veces por semana por 30 minutos. Las medidas del ángulo de la rodilla de las ratas se realizó mediante el uso de Dartfish (análisis biomecánico que traza la trayectoria de los segmentos marcados). El ángulo de la rodilla se midió en un pre test y en un post test. En la OA se produce rigidez de las articulaciones, lo que observaron en la disminución del ángulo de la rodilla de ratas con MIA y ejercicio sin cambios de velocidad, por el contrario, observaron un aumento del ángulo de rodilla en las ratas con MIA y cambios de velocidad de la caminata. Indicando que la rigidez de las articulaciones sometidas al ejercicio con cambios de velocidad fue menos progresiva.

Mohammadi et al. (2013) desarrollaron un estudio que tuvo como objetivo investigar el efecto del ejercicio moderado en cinta rodante por cuatro semanas, sobre la osteoartritis de rodilla en ratas, la cual se indujo mediante MIA. Se dividieron en 3 grupos: control intacto, MIA, y MIA con entrenamiento, Se siguió un programa de ejercicios de intensidad moderada durante 28 días, luego se realizó una evaluación histológica de la articulación de la rodilla por escala OARSI. Los resultados demostraron que el ejercicio moderado durante 4 semanas podría tratar los síntomas de la OA sobre la articulación de la rodilla por lo cual se concluye el que ejercicio moderado en cinta rodante ejerce una influencia beneficiosa sobre el cartílago articular.

Musumeci et al. (2015), en su estudio, utilizaron el ejercicio físico en ratas jóvenes, adultas y ancianas sanas, para evaluar la expresión de la lubricina como un nuevo biomarcador de la senescencia de condrocitos; los resultados sugieren que el ejercicio físico moderado, la carga articular normal y la estimulación mecánica en ratas de edad avanzada mejoran la lubricación y previenen la degeneración del cartílago, promoviendo la síntesis de lubricina en líquido sinovial, en comparación con las ratas adultas sedentarias; La actividad física aumenta la movilidad articular, por lo tanto, tanto el líquido sinovial como los condrocitos expresan más lubricina, lo que resulta en una mejor lubricación de las superficies articulares. Este mecanismo de lubricación protectora ayuda a prevenir la aparición de la OA en el envejecimiento; los resultados también apoyan la hipótesis de que la actividad física también podría usarse en el envejecimiento como un tratamiento terapéutico natural para las enfermedades del cartílago para prevenir la OA y limitar los efectos nocivos de la senescencia de condrocitos. 
Además de ejercicios en cintra rodante, se realizaron estudios con protocolo de ejercicio acuático. Al-Hashem et al. (2017) realizaron un estudio que demuestra que el ejercicio de natación puede resguardar el cartílago articular. Para lo cual utilizaron ratas Sprague Dawley a las que se les indujo la OA a través de la diabetes miellitus. Se les aplico un protocolo de ejercicio de natación de $30 \mathrm{~min}, 3$ veces a la semana, por un periodo de 8 semanas. Se realizaron análisis de microscopía electrónica de barrido para evaluar cortes de las articulaciones de rodilla. Se resolvió que el ejercicio de natación inhibe la peroxidación lipídica y aumenta el SOD (súper oxido dismutasa). Además el ejercicio inhibe el TNFa inducido por T1DM. Se comprueba que el ejercicio es preventivo y disminuye el riego de desarrollar OA en insulinos dependientes.

Assis et al. (2018) tuvieron como objetivo comparar los efectos de la fotobiomodulación (PBM) asociados al ejercicio acuático y aeróbico sobre el cartílago articular de ratas con osteoartritis (OA). Las ratas Wistar fueron divididas en 5 grupos con diferentes protocolos de ejercicios aeróbicos y acuáticos, algunos asociados al grupo de fotobiomodulación. Los ejercicios se realizaron 3 días a la semana, durante 8 semanas. Se realizaron evaluaciones morfológicas y de inmunoexpresion de IL-10, TGF-B y colágenos tipo I Y II del cartílago articular. Se obtuvo como resultado que todos los grupos que cumplieron el protocolo de ejercicio y PBM mostraban signos menos intensos de degradación. También las ratas con ejercicio aeróbico y acuático asociados con la PBM o no, mostraron un aumento de la IL-10 en comparación al equipo de control. Además presentaron una expresión de TGF - B superior al grupo de control. Se sugiere que la fotobiomodulación asociada a ejercicios aeróbicos y acuáticos es eficaz para mediar efectos condroprotectores que mantienen la integridad del tejido articular en las rodillas de ratas con OA.

Por otro lado, investigaciones demostraron que el ejercicio ayuda a disminuir el dolor producido por OA. Park et al. (2015) investigaron los efectos del ejercicio sobre la expresión del factor de crecimiento nervioso en células nerviosas de médula espinal dañadas, debido a las señales de dolor que son un síntoma importante de la osteoartritis. Utilizaron ratas Sprague-Dawley adultas y la inducción de osteoartritis fue por MIA, se dividieron al azar en 4 grupos: grupo control inyección de solución salina, grupo MIA sin ejercicio (SG), grupo MIA de 3 semanas (CG), grupo MIA 7 semanas y grupo MIA con 4 semanas de entrenamiento (tres semanas post inyección de MIA). El grupo de ejercicios se sometió a un programa de entrenamiento de 4 semanas en una cinta rodante durante 5 días, por treinta minutos diarios. Extrajeron la médula espinal y midieron la expresión del factor de crecimiento nervioso (NGF) mediante inmunohistoquímica, el cuál se vió aumentado en el grupo de ejercicio comparado con el grupo control y el grupo de MIA sin ejercicio. Concluyeron que la expresión aumentada del factor de crecimiento nervioso en la medula espinal inducido por la estimulación del ejercicio, puede ser efectiva en tratamientos de dolor crónico. Este tratamiento no solo contribuirá a mejorar la función de la articulación de pacientes con dolor crónico, sino que también su calidad de vida. Además, Park et al. (2016), investigaron con el mismo protocolo, la expersión de GAP-43, que juega un rol importante en la regeneración y recuperación de células nerviosas de la médula espinal dañadas por la OA crónica. Observando que el ejercicio aumentaba la expresión de GAP-43 en la médula espinal para promover la recuperación de las células nerviosas de la médula espinal.

Allen et al. (2017) evaluaron los efectos del ejercicio sobre el dolor de la OA avanzada, resistente a los antiinflamatorios no esteroidales (AINE). Utilizaron MIA para producir OA. El ejercicio en corredora por cuatro semanas, demostró reducir la hipersensibilidad táctil y asimetría de la distribución del peso de los miembros. Apoyando la conclusión de que el ejercicio induce alivio del dolor en OA avanzada, resistente a los AINE, probablemente a través de un aumento de la señalización opioide endógena. Además, el ejercicio en cinta rodante bloqueó la pérdida ósea inducida por MIA en este modelo, lo que indicó que el ejercicio tiene un potencial efecto estabilizador óseo sobre la articulación con $\mathrm{OA}$.

Galois et al. (2004) investigaron la influencia de ejercicio suave, moderado e intenso, sobre modelo experimental de OA, producido por corte de ligamento cruzado anterior, evaluando apoptosis a nivel de condrocitos, estableciendo que el ejercicio suave y moderado es beneficioso para el cartílago, mientras que el ejercicio intenso, suprime el factor condroprotector.

Dentro de los artículos que mostraron efectos nocivos del ejercicio encontramos a Li et al. (2015), que investigaron el impacto del ejercicio en alta intensidad exhaustivo sobre la expresión del óxido nítrico, malonodialdehído y la superóxido dismutasa, en ratas con OA de rodilla. El protocolo de ejercicio consistía en hacer correr a las ratas en una corredora a una velocidad de $15 \mathrm{~m} / \mathrm{min}$ durante $15 \mathrm{mi}$ nutos, luego la velocidad se aumentaba gradualmente hasta un máximo de $35 \mathrm{~m} / \mathrm{min}$ hasta que las ratas estuvieran exhaustas. En el desarrollo de la $\mathrm{OA}$ se ha demostrado que niveles de citoquinas, radicales libres, óxido nítrico y malondialdehido tienen estrecha relación con la degradación del cartílago articular. Los resultados de este estudio, demostraron que con el aumento de la intensidad del ejercicio, la actividad de la superóxido dismutasa en las rodillas 
de rata con OA disminuyó gradualmente, mientras que los niveles de óxido nítrico, malonodialdehído aumentaron gradualmente, por lo que mientras mayor sea la intensidad del ejercicio, mayor será el impacto en las rodillas con OA.

Ni et al. (2016) compararon los cambios patológicos en el cartílago derivado de la OA en ratas desarrollada por la inmovilización articular o por entrenamiento en corredora exhaustivo, para entender mejor los respectivos mecanismos patológicos. Las características histológicas y expresión genética de los proteoglicanos y colágeno tipo II, sugieren que ambos métodos: inmovilización de la rodilla y el correr de manera extenuante, podrían llevar a cambios degenerativos del cartílago, pero a diferentes etapas del proceso degenerativo. La evaluación de la degradación del cartílago, según la escala de MANKIN, mostró una degradación moderada del cartílago luego de 8 semanas de correr de manera extenuada, sin embargo, en el grupo que fue inmovilizado, se observó un daño más severo del cartílago.

Moshtagh et al. (2018) investigaron las alteraciones tempranas del hueso y el cartílago provocadas por un protocolo de ejercicio intenso y continuo, en ratas Wistar. La hipótesis de este estudio, era que un pre-entrenamiento (de baja intensidad) al protocolo de ejercicio intenso continuo, podría proteger a los animales de la degradación del cartílago. Midieron con escala OARSI, pero no pudieron categorizar en la escala los pequeños cambios iniciales observados, ya que la escala de puntuación para el cartílago y morfología de los condrocitos no es muy precisa. No pudieron concluir si los animales ejercitados aún estaban dentro de los límites de un cartílago saludable o estaban entrando a un estadio temprano de OA. Pero si concluyeron, que el protocolo de pre-entrenamiento con aumento de intensidad progresiva del ejercicio, no demostró ser un factor protector para el cartílago articular.

Cormier et al. caracterizaron los efectos del monoiodo acetato de sodio (MIA), sobre la asimetría de la distribución del peso de los miembros posteriores de ratas sedentarias y con acceso a una rueda corredora. Se les inyectó $3.2 \mathrm{mg}$ de MIA en la rodilla izquierda, fueron divididas en dos grupos: con acceso voluntario a la rueda corredora durante 24 horas al día por 42 días consecutivos; el grupo sedentario, sin acceso a rueda corredora. Se midió la asimetría a los 3, 7 y 21 días post inyección. El grupo sedentario, sólo mostró diferencias de asimetría en los días 3 y 7 , en cambio, el grupo con ejercicio, mostraron asimetría del peso en las tres ocasiones. Para determinar los potenciales mecanismos causales detrás de las diferencias entre las ratas ejercitadas y sedentarias, se realizó un análisis de imágenes microCT ex vivo para evaluar los cambios arquitectónicos en el miembro posterior del fémur distal / tibia proximal y la rodilla de las ratas. Los resultados indicaron que las ratas ejercitadas e inyectadas con MIA tenían una mayor pérdida relativa del volumen subcondral total lateral comparado con los sedentarios inyectados con MIA. Mostrando que las ratas que corrieron voluntariamente, exacerbaron los efectos de la OA inducida por MIA en ambas mediciones de comportamiento y biología ósea.

Tomazoni et al. (2017) evaluaron y compararon el efecto de terapia de Fotobiomodulación, ejercicio físico y antiinflamatorio tópico no esteroideo, utilizados en el proceso inflamatorio, utilizados solos o en diferentes combinaciones. La OA fue inducida por la inyección de papaína en la articulación de rodilla en ratas. El tratamiento comenzó 21 días post inyección con antiinflamatorio y/o ejercicio y/ o fotobiomodulación, por tres veces a la semana durante 8 semanas, con un total de 24 sesiones de terapia. Se realizaron análisis de reacción en cadena de polimerasa en tiempo real (PCR) para evaluar la expresión génica de Interleuquina (IL)-1 $\beta$, IL-6 y factor de tumor de necrosis alfa (TNF- $\alpha$ ) por test de ELISA. Los resultados obtenidos en este estudio, mostraron que el tratamiento con fotobiomodulación es más efectivo para modular el proceso inflamatorio de la OA, comparado con las otras terapias probadas.

Zhou et al. (2014) desarrollaron una investigación que tenía por objetivo medir la expresión del receptor potencial transitorio del canal catión del V5 (TRPV5) en las células del cartílago articular bajo diferentes cargas de ejercicio y analizar la relación de TRPV5 en la condrogénesis. Para esto se utilizaron ratas Sprague Dawley, a las cuales se les indujo la OA a través de MIA y fueron sometidas a realizar ejercicio en cinta rodante durante 30 minutos dos veces al día durante 8 semanas. Luego de las 8 semanas se realizó una clasificación macroscópica con las lesiones del cartílago de acuerdo con el Outerbridge estándar. La medición de la expresión de TRPV5 se realizó después de la carga de ejercicio para asociarlo con patologías clínicas a través de inmunohistoquímica. Se confirmó que la expresión de TRPV5 se expresó en todo el tejido óseo y articular y su nivel de expresión, dependía del nivel de la carga del hueso y de la articulación. Concluyeron que la expresión de TRPV5, podría jugar un rol importante en los procesos de formación del tejido óseo y cartilaginoso.

Franciozi et al. (2013) plantearon la hipótesis de que uno de los factores que puede ser predeterminante para la osteoartritis, es la carrera vigorosa. Para su ejecución se trabajó con ratas Wistar que fueron sometidas a ejercicio, el cual se efectuó a una intensidad moderada en cinta rodante por un periodo de 12 semanas, para que las ratas mejoraran su resistencia y pudieran culminar el entrenamiento con una carrera de ultra resistencia correspondiente al $60 \%$ de su 
velocidad máxima. Luego realizaron análisis histomorfométricos, histomorfológicos e inmunohistoquímicos para evaluar la muerte celular. Las ratas entrenadas mostraron mayor número de condrocitos y niveles altamente significativos de caspasa - 3 , que se relaciona directamente con la apoptosis y la inflamación de citoquinas IL-1a t TNF-a, además de un mayor grado de OA según la escala OARSI. En conclusión las ratas con régimen de carrera muestran predisposición a la OA. Además de una disminución significativa de GAGs sulfatados y ácido hialurónico que son componentes de la matriz extracelular. Concluyendo que las ratas sometidas al ejercicio de carrera extenuante, mostraron características moleculares e histológicas que están presentes en la OA.

Siebelt et al. (2014b) evaluaron si el ejercicio moderado podría tener beneficios o si aumenta la susceptibilidad a la degeneración del cartílago. Utilizaron ratas Wistar a las cuales se les administró inyecciones intra-articulares de papaína para reducir el contenido glicosaminoglicanos sulfatados (sGAG) del cartílago de las rodillas. Luego, a la mitad del grupo de estudio, se aplicó un protocolo de ejercicio para proteger la degradación del cartílago en donde recorrían un total de $15 \mathrm{~km}$ durante 6 semanas. Se realizaban $5 \mathrm{~min}$ a $20 \mathrm{~cm} / \mathrm{s}$ de calentamiento y luego $25 \mathrm{~min}$ a $35 \mathrm{~cm} / \mathrm{s}$ trabajando en cinta rodante a $25 \%$ de su capacidad durante las sesiones. Se analizaron las muestras con micro CT, histología y se midió el grosor del cartílago. Como resultado las ratas ejercitadas mostraron un aumento en la degradación de la matriz del cartílago, formaciones óseas escleróticas y aumento en la formación de osteofitos y activación de macrófagos. Concluyeron que el ejercicio moderado no protegió contra el desarrollo de la osteoartritis inducida por papaína y dió lugar a la esclerosis subcondral pronunciada, acelerando la progresión de la OA.

Dentro de la revisión se encontraron artículos donde no se obtuvieron resultados concretos sobre los efectos del ejercicio en la articulación con $\mathrm{OA}$, con resultados buenos y malos para el tratamiento de la OA con ejercicio.

Yamaguchi et al. (2013) estudiaron si los niveles de biomarcadores séricos, reflejaban la progresión de la OA, inducida por diferentes niveles de ejercicio. Utilizaron el ejercicio en corredora para evaluar su efecto sobre OA en rodillas de rata tras cortar el ligamento cruzado anterior. Con el fin de evaluar niveles de biomarcadores séricos colágeno tipo II (C2C) y procolageno II (CPII) y estadío de la enfermedad utilizando OARSI. El estudio fue realizado en ratas Wistar, utilizaron dos protocolos de ejercicio a una misma velocidad $(18 \mathrm{~m} / \mathrm{min})$, tres veces a la semana por: moderado $30 \mathrm{~min} /$ día y alta intensidad $60 \mathrm{~min} /$ día durante 2 o 4 semanas. El ejercicio intenso provocó una degradación rápida del cartílago ( 2 semanas post cirugía) y el grupo de entrenamiento moderado no mostró una degradación seria del cartílago, pero si presentaron pequeños daños (como pérdida de proteoglicanos y pérdida de superficie articular ininterrumpida). En la escala OARSI, a las dos semanas, no se observaron diferencias entre el control y el grupo de alta intensidad y el grupo de entrenamiento moderado, a las 4 semanas los niveles de C2C/CPII que se encontraría deterioro en la escala OARSI, pero la evaluación con OARSI difirió significativamente entre los grupos de ejercicio moderado y alta intensidad, concordando con los niveles de $\mathrm{C} 2 \mathrm{C} / \mathrm{CPII}$ que se vieron aumentados en el grupo de ejercicio de alta intensidad.

Lee et al. propusieron un nuevo sistema de evaluación histopatológica, para la evaluación temprana de la OA causada por ejercicio y así para simular la enfermedad humana. Para ello se sometieron ratas Wistar a una intervención con ejercicio en cinta rodante con variaciones en el ángulo y la inclinación de los carriles. Se utilizó el protocolo de ejercicio de caminata continua con una frecuencia de una hora al día, durante 6,8 y 10 semanas. Se usó el sistema de puntuación Mankin para determinar el nivel de la OA agrupándola en leve, moderada o severa. Además del sistema OARSI el cual se empleó de acuerdo al cartílago femoral y tibial. El análisis buscaba la asociación entre las puntuaciones histológicas y la duración del ejercicio. Finalmente se determinó que la OA inducida por ejercicio en cinta rodante se puede utilizar para el estudio de esta patogenia. También que el sistema de puntuación MMS es más útil en la evaluación de OA que el de la OARSI.

Appleton et al. (2007a) desarrollaron un estudio con el propósito de analizar de manera integral la patogénesis de la OA. Para esto se utilizaron ratas Sprague-Dawley a las que se les indujo la OA para evaluar tratamientos secundarios. También se vieron los efecto producidos al caminar sobre un cilindro giratorio, con esta desestabilización inducida se analizó la degradación del cartílago articular, cambios subcondrales, y perdida de hueso subcondral. La movilización del ejercicio forzó la OA y su progresión, se observó perdida de proteoglicanos, además de aumento de condrocitos durante la hipertrofia en la patogenia. En el estudio también se incluyó ejercicio de mediana intensidad (FM) que correspondía a caminata sobre un cilindro $30 \mathrm{~min}$ 3 veces a la semana, obligando a la máxima extensión y flexión de la rodilla. La FM aceleraba a OA y el ejercicios con cargas repetitivas es perjudicial para las articulaciones desestabilizadas.

Tomazoni et al. (2016) se plantearon como objetivo de estudio, evaluar y comparar los efectos de diversos tratamientos de la OA inducida por papaína: con medicamento antiinflamatorio no esteroidal tópico (AEINE), actividad 
física y fotobiomodulación. Para el desarrollo del experimento se dividió el grupo en 6, dentro de los cuales algunos recibían tratamiento con ejercicio acuático, tratamiento de diclofenaco sódico y otros con fotobiomodulación. Todos los grupos experimentales comenzaron 21 días después de la inyección de papaína y se realizaron 24 sesiones de terapia. Los grupos con ejercicio realizaban $100 \times 50 \times 75 \mathrm{~cm}^{3}$ de natación. De acuerdo a los resultados del estudio, los autores concluyeron que el tratamiento más efectivo para reducir la expresión de citoquinas pro -inflamatorias, fue el tratamiento con fotobiomodulación.

Ni et al. (2011) estudiaron el efecto de la inyección intraarticular de inhibidor de mataloprotenasa de matriz (MMP) - 3, para evaluar cuál es el su rol en la degradación del cartílago articular en OA inducida por carrera excesiva. Para esta investigación se utilizaron ratas Wistar que fueron dividas en cuatro grupos: sedentario de control (SED), entrenamiento de alta intensidad (HIR), entrenamiento de alta intensidad + dosis baja de inhibidor MMP-3 (HIRI1) y entrenamiento de alta intensidad + dosis alta de Inhibidor de MMP-3 I (HIRI2). Las ratas realizaron ejercicio $1 \mathrm{~h} /$ día a la frecuencia 5 días / semana, la intensidad fue de $25 \mathrm{~m} /$ minuto e inclinación $12^{\circ}$ (85\% VO2 máx.). Se hicieron análisis histológicos para evaluar características del cartílago, inmunohistoquímicos y evaluación de muestras de sangre por test ELISA para medir niveles de MMP-3.

El cartílago mostró cambios característicos de OA luego de 6 semanas de carrera de alta intensidad, en comparación con el grupo SED, se encontraron disminuciones significativas en los glucosaminoglucanos (GAG) y el contenido de colágeno en el grupo de carrera de alta intensidad, lo que correspondió a un aumento significativo en el nivel sérico de MMP-3, la actividad del cartílago MMP-3 y la expresión génica. Sin embargo, dicho proceso degenerativo se retrasó considerablemente mediante la inyección intraarticular del inhibidor de MMP-3 a dosis más altas. Concluyendo que la carrera de alta intensidad puede producir degradación en el cartílago articular, pero con la inyección del inhibidor MMP-3 se puede desarrollar un efecto condroprotector, generando cambios catabólicos bajo esa condición mecánica, siendo una opción eficaz para retardar la generación de OA.

Yang et al. (2019) evaluaron los efectos y mecanismos del estrés mecánico sobre el cartílago articular con OA inducida por MIA, por medio de diferentes protocolos de ejercicios aplicados en ratas Sprague-Dawley. Para la ejecución de los ejercicios dividieron a las ratas en ocho grupos: Cuatro grupos sanos: CG grupo de control (sin ejercicio); CL (con ejercicio de baja intensidad, $30 \mathrm{~min}$ al día), CM (ejercicio de mediana intensidad, $60 \mathrm{~min}$ al día), $\mathrm{CH}$ (ejercicios de alta in- tensidad, 90 min al día) y cuatro grupos con Osteoartritis inducida por MIA:OAG (sin ejercicio), OAL (ejercicio de baja intensidad), OAM (ejercicio de mediana intensidad) y $\mathrm{OAH}$ (ejercicios de alta intensidad). Luego de cuatro semanas ejercitándose en cinta rodante evaluaron las lesiones articulares y de acuerdo a los resultados de las puntuaciones histológicas se encontró que no habían diferencias entre el grupos CM y CG, pero la OAM presentaba efectos terapéuticos a diferencia del OAG. Por otro lado los grupos CH Y OAH tenían más daños en el cartílago debido al protocolo de ejercicio. Concluyeron que no hubo efectos terapéuticos por causa del estrés mecánico y la estimulación excesiva con ejercicio de alta intensidad, sólo causó daño. Sin embargo el estrés moderado podría reducir la sensibilización a la respuesta inflamatoria del cartílago articular y los condrocitos por la vía de señalización de AMPK / NF kb.

Iijima et al. (2017) se plantearon como objetivo de estudio, determinar, si la caminata en cinta rodante previene la progresión de los cambios en el hueso subcondral y el cartílago, provocados por OA postraumática. Utilizaron ratas Wistar machos que sometieron a cirugía de desestabilización del menisco medial en la rodilla derecha, fueron asignados aleatoriamente en dos grupos: sedentario y de caminata. El protocolo de ejercicios se inició desde el segundo día a 4 semanas post cirugía. Utilizaron micro CT, tomogtrafía, análisis histológico e inmunohistoquímica para la evaluación del hueso subcondral.

Los resultados que obtuvieron, demostraron que la caminata pudo prevenir la progresión de lesiones inducidas por la cirugía en el cartílago y el hueso subcondral, aumentando la expresión de la proteína morfogénica ósea (BMP)2 y-6, en la zona superficial de condrocitos y en c\{elulas de linaje óseo, incluyendo osteoblastos. Además, el aumento de expresión de BMP-2 y-6 inducida por la caminata, varió respecto al tiempo transcurrido post cirugía para comenzar el ejercicio, demostrando ser más efectiva en el aumento de BMPs, la intervención de la caminata a las 4 semanas post cirugía. Por otra parte, Appleton et al. (2007b) determinaron que la articulación de la rodilla desestabilizada es susceptible a una mayor degeneración del cartílago cuando se somete a ejercicio de baja intensidad o bajo impacto.

Estudios previos también han investigado la relación del ejercicio con la composición del cartílago. Bricca et al. (2017) realizaron una revisión sistemática de ensayos controlados aleatorios (ECA) para investigar los efectos de una dosis diaria de ejercicio sobre el grosor y la composición del cartílago articular. Para el desarrollo del estudio se hizo una síntesis narrativa de la dosis diaria del ejercicio en el cartílago de rodilla, colágeno y espesor. Se evaluaron estudios con datos suficientes para realizar un meta análisis de 
modelos con efectos aleatorios. Se usaron 29 ECA que tenían 64 comparaciones. En el protocolo de ejercicio en dosis bajas 21/25 informaron efecto reducido o nulo en el cartílago. El ejercicio en dosis moderada las 12 comparaciones informaron un efecto no mayor. El ejercicio en dosis alta 19/27 mostraron un efecto reducido. Además de 14 estudios que analizaron grosor de cartílago en dosis baja de ejercicio no se observaron efectos, en dosis moderada el engrosamiento fue grande pero no significativo y en altas dosis de ejercicio, se vio adelgazamiento del cartílago. Se concluyó que las dosis bajas de ejercicios no mostraban resultados concluyentes, la dosis diaria de ejercicio de alta intensidad puede generar efectos negativos y una dosis moderada puede tener efectos positivos en la composición de la matriz del cartílago.

\section{CONCLUSIÓN}

Esta revisión, refleja los estudios que se han realizado, sobre los efectos del ejercicio en la osteoartritis de rata, aunque aún hay resultados controversiales sobre si el ejercicio es beneficiosos o no para ratas que padecen osteoarthritis, ya sea inducida por droga o por cirugía, los estudios más recientes, demuestran que el ejercicio aeróbico moderado es beneficioso para ser utilizado como tratamiento de la OA. Sin embargo aún queda dilucidar la "dosis" o intensidad exacta de ejercicio, ya que el ejercicio excesivo, puede provocar más daño a la articulación con OA. En este sentido, fututos estudios deberían clarificar la intensidad recomendada de ejercicio, tomando en cuenta el estadío de avance de la OA.

VARGAS, C. A.; SANDOVAL, K. A.; SALVO, C. A.; DEL SOL, M. \& OTTONE, N. E. Exercise in rats osteoarthritis. Morphological aspects and literature review. Int. J. Morphol., 38(2):481-491, 2019

SUMMARY: Osteoarthritis (OA) is a chronic, degenerative, and very disabling disease that gradually and progressively destroys articular cartilage in various regions: knees, hips, shoulders, hands, ankles and spine. In this sense, exercise has been described as the most recommended non-pharmacological intervention for patients with OA. Regular exercise is considered an integral component of the healthy lifestyle. However, its effect on cartilage remains the subject of debate and speculation, as well as the relationship between exercise and the development of OA. Some animal model studies suggest that exercise may be beneficial for cartilage health, while others demonstrate its harmful effect. A general explanation for these inconsistent results is that running at moderate intensity has beneficial effects, while running "vigorously" or "strenuously" leads to a harmful effect. The aim of this work was to make a literature review about the effects of exercise on cartilage, especially focused on experimental animal models with rats.

KEY WORDS: Osteoarthritis; Exercise; Rats; Morphology.

\section{REFERENCIAS BIBLIOGRÁFICAS}

Al-Hashem, F.; El Karib, A. O.; Bin-Jaliah, I.; Dallak, M.; Sakr., H. F.; Eid, R. A.; Zaki, M. S. A.; Al-Shamsi, M.; Haidara, M. A. \& Al-Ani, B. Exercise protects against insulin-dependent diabetes-induced osteoarthritis in rats: a scanning electron microscopy study. Ultrastruct. Pathol., 41(3):252-7, 2017.

Allen, J.; Imbert, I.; Havelin, J.; Henderson, T.; Stevenson, G.; Liaw, L. \& King, T. Effects of Treadmill Exercise on Advanced Osteoarthritis Pain in Rats. Arthritis Rheumatol., 69(7):1407-17, 2017.

Appleton, C. T. G.; McErlain, D. D.; Henry, J. L.; Holdsworth, D. W. \& Beier, F. Molecular and histological analysis of a new rat model of experimental knee osteoarthritis. Ann. N. Y. Acad. Sci., 1117:165-74, 2007b.

Appleton, C. T. G.; McErlain, D. D.; Pitelka, V.; Schwartz, N.; Bernier, S. M.; Henry, J. L.; Holdsworth, D. W. \& Beier, F. Forced mobilization accelerates pathogenesis: characterization of a preclinical surgical model of osteoarthritis. Arthritis Res. Ther, 9(1):R13, 2007a.

Assis, L.; Almeida, T.; Milares, L. P.; dos Passos, N.; Araújo, B.; Bublitz, C.; Veronez, S. \& Renno, A. C. M. Musculoskeletal atrophy in an experimental model of knee osteoarthritis: the effects of exercise training and low-level laser therapy. Am. J. Phys. Med. Rehabil., 94(8):609-16, 2015.

Assis, L.; Milares, L. P.; Almeida, T.; Tim, C.; Magri, A.; Fernandes, K. R.; Medalha, C. \& Muniz Renno, A. C. Aerobic exercise training and low-level laser therapy modulate inflammatory response and degenerative process in an experimental model of knee osteoarthritis in rats. Osteoarthritis Cartilage, 24(1):169-77, 2016.

Assis, L.; Tim, C.; Magri, A.; Fernandes, K. R.; Vassão, P. G. \& Renno, A. C. M. Interleukin-10 and collagen type II immunoexpression are modulated by photobiomodulation associated to aerobic and aquatic exercises in an experimental model of osteoarthritis. Lasers Med. Sci., 33(9):1875-82, 2018.

Åstrand, P. O. \& Rodahl, K. Textbook of Work Physiology. Physiological Bases of Exercise. $3^{\mathrm{a}}$ ed. New York, McGraw-Hill, 1986.

Bedford, T. G.; Tipton, C. M.; Wilson, N. C.; Oppliger, R. A. \& Gisolfi, C. V. Maximum oxygen consumption of rats and its changes with various experimental procedures. J. Appl. Physiol. Respir. Environ. Exerc. Physiol., 47(6): 1278-83, 1979.

Bendele, A. M. Animal models of osteoarthritis. J. Musculoskelet. Neuronal Interact., 1(4):363-76, 2001.

Blazek, A. D.; Nam, J.; Gupta, R.; Pradhan, M.; Perera, P.; Weisleder, N. L.; Hewett, T. E.; Chaudhari, A. M.; Lee, B. S.; Leblebicioglu, B.; et al. Exercisedriven metabolic pathways in healthy cartilage. Osteoarthritis Cartilage, 24(7):1210-22, 2016.

Bricca, A.; Juhl, C. B.; Grodzinsky, A. J. \& Roos, E. M. Impact of a Daily Exercise Dose on Knee Joint Cartilage - A Systematic Review and Meta-Analysis of Randomized Controlled Trials in Healthy Animals. Osteoarthritis Cartilage, 25(8):1223-37, 2017

Castrogiovanni, P.; Di Rosa, M.; Ravalli, S.; Castorina, A.; Guglielmino, C.; Imbesi, R.; Vecchio, M.; Drago, F.; Szychlinska, M. A. \& Musumeci, G. Moderate physical activity as a prevention method for knee osteoarthritis and the role of synoviocytes as biological key. Int. J. Mol. Sci., 20(3), 2019.

Cifuentes, D. J.; Rocha, L. G.; Silva, L. A.; Brito, A. C.; Rueff-Barroso, C. R.; Porto, L. C. \& Pinho, R. A. Decrease in oxidative stress and histological changes induced by physical exercise calibrated in rats with osteoarthritis induced by monosodium iodoacetate. Osteoarthritis Cartilage, 18(8):108895, 2010.

Cormier, J.; Cone, K.; Lanpher, J.; Kinens, A.; Henderson, T.; Liaw, L.; Bilsky, E. J.; King, T.; Rosen, C. J. \& Stevenson, G. W. Exercise reverses painrelated weight asymmetry and differentially modulates trabecular bone microarchitecture in a rat model of osteoarthritis. Life Sci., 180:51-9, 2017.

Franciozi, C. E. S.; Tarini, V. A. F.; Reginato, R. D.; Gonçalves, P. R. S.; Medeiros, V. P.; Ferretti, M.; Dreyfuss, J. L.; Nader, H. B. \& Faloppa, F. Gradual strenuous running regimen predisposes to osteoarthritis due to cartilage cell death and altered levels of glycosaminoglycans. Osteoarthritis Cartilage, 21(7):965-72, 2013.

Galois, L.; Etienne, S.; Grossin, L.; Watrin-Pinzano, A.; Cournil-Henrionnet, C.; Loeuille, D.; Netter, P.; Mainard, D. \& Gillet, P. Dose-response relationship for exercise on severity of experimental osteoarthritis in rats: a pilot study. Osteoarthritis Cartilage, 12(10):779-86, 2004. 
Hansen, P.; English, M. \& Willick, S. E. Does running cause osteoarthritis in the hip or knee? PM R, 4(5 Suppl.):S117-21, 2012.

Hong, Y.; Kim, H.; Lee, Y.; Lee, S.; Kim, K.; Jin, Y.; Lee, S. R.; Chang, K. T. \& Hong, Y. Salutary effects of melatonin combined with treadmill exercise on cartilage damage. J. Pineal Res., 57(1):53-66, 2014.

Iijima, H.; Ito, A.; Nagai, M.; Tajino, J.; Yamaguchi, S.; Kiyan, W.; Nakahata, A.; Zhang, J.; Wang, T.; Aoyama, T.; et al. Physiological exercise loading suppresses post-traumatic osteoarthritis progression via an increase in bone morphogenetic proteins expression in an experimental rat knee model. Osteoarthritis Cartilage, 25(6):964-75, 2017.

Kiviranta, I.; Tammi, M.; Jurvelin, J.; Säämänen, A. M. \& Helminen, H. J. Moderate running exercise augments glycosaminoglycans and thickness of articular cartilage in the knee joint of young Beagle dogs. J. Orthop. Res., 6(2): 188-95, 1988

Knobloch, T. J.; Madhavan, S.; Nam, J.; Agarwal Jr., S. \& Agarwal, S. Regulation of chondrocytic gene expression by biomechanical signals. Crit. Rev. Eukaryot. Gene Expr., 18(2):139-50, 2008.

Kuyinu, E. L.; Narayanan, G.; Nair, L. S. \& Laurencin, C. T. Animal models of osteoarthritis: classification, update, and measurement of outcomes. $J$. Orthop. Surg. Res., 11:19, 2016.

Lavalle Montalvo, C. Osteoartritis. En: Seminarios sobre Medicina y Salud. Ciudad de México, Universidad Nacional Autónoma de México, 2010.

Lee, Y. J.; Park, J. A.; Yang, S. H.; Kim, K. Y.; Kim, B. K.; Lee, E. Y.; Lee, E. B.; Seo, J. W.; Echtermeyer, F.; Pap, T. \& Song, Y. W. Evaluation of osteoarthritis induced by treadmill-running exercise using the modified Mankin and the New OARSI assessment system. Rheumatol. Int., 31(12):1571-6, 2011.

Li, X. D.; Sun, G. F.; Zhu, W. B. \& Wang, Y. H. Effects of high intensity exhaustive exercise on SOD, MDA, and NO levels in rats with knee osteoarthritis. Genet. Mol. Res., 14(4):12367-76, 2015.

Link, T. M. \& Li, X. Bone marrow changes in osteoarthritis. Semin. Musculoskelet. Radiol., 15(3):238-46, 2011.

Milner, P. I.; Fairfax, T. P. A.; Browning, J. A.; Wilkins, R. J. \& Gibson, J. S. The effect of $\mathrm{O} 2$ tension on $\mathrm{pH}$ homeostasis in equine articular chondrocytes. Arthritis Rheum., 54(11):3523-32, 2006.

Mohammadi, M. F.; Moghaddam, A. H. \& Mirkarimpur, H. The effects of a moderate exercise program on knee osteoarthritis in male Wistar rats. Iran. J. Basic Med. Sci., 16(5):683-8, 2013.

Moshtagh, P. R.; Korthagen, N. M.; Plomp, S. G.; Pouran, B.; Castelein, R. M.; Zadpoor, A. A. \& Weinans, H. Early signs of bone and cartilage changes induced by treadmill exercise in rats. JBMR Plus, 2(3):134-42, 2018.

Murray, R. C.; Vedi, S.; Birch, H. L.; Lakhani, K. H. \& Goodship, A. E. Subchondral bone thickness, hardness and remodelling are influenced by short-term exercise in a site-specific manner. J. Orthop. Res., 19(6):103542, 2001.

Musumeci, G.; Castrogiovanni, P.; Trovato, F. M.; Imbesi, R.; Giunta, S.; Szychlinska, M. A.; Loreto, C.; Castorina, S. \& Mobasheri, A. Physical activity ameliorates cartilage degeneration in a rat model of aging: a study on lubricin expression. Scand. J. Med. Sci. Sports, 25(2):e222-30, 2015.

Na, S. S.; Kim, S. G.; Yong, M. S. \& Hwangbo, G. Study of treadmill exercise effect on rats with osteoarthritis using proteomic analysis. J. Phys. Ther Sci., 26(4):487-90, 2014.

Nam, C. W.; Kim, K. \& Na, S. S. The effect of changing condition of walking speed on the knee angle of rats with osteoarthritis. J. Phys. Ther. Sci. 29(8):1387-9, 2017.

Ni, G. X.; Lei, L. \& Zhou, Y. Z. Intensity-dependent effect of treadmill running on lubricin metabolism of rat articular cartilage. Arthritis Res. Ther. 14(6):R256, 2012.

Ni, G. X.; Liu, S. Y.; Lei, L.; Li, Z.; Zhou, Y.Z. \& Zhan, L. Q. Intensity-dependent effect of treadmill running on knee articular cartilage in a rat model. Biomed Res. Int., 2013:172392, 2013.

Ni, G. X.; Zhan, L. Q.; Gao, M. Q.; Lei, L.; Zhou, Y. Z. \& Pan, Y. X. Matrix metalloproteinase- 3 inhibitor retards treadmill running-induced cartilage degradation in rats. Arthritis Res. Ther., 13(6):R192, 2011.

Ni, G. X.; Zhou, Y. Z.; Chen, W.; Xu, L.; Li, Z.; Liu, S. Y.; Lei., L. \& Zhan, L. Q. Different Responses of Articular Cartilage to Strenuous Running and Joint Immobilization. Connect. Tissue Res., 57(2):143-51, 2016.

Otterness, I. G.; Eskra, J. D.; Bliven, M. L.; Shay, A. K.; Pelletier, J. P. \& Milici, A. J. Exercise protects against articular cartilage degeneration in the hamster. Arthritis Rheum., 41(11):2068-76, 1998.
Park, S. J.; Jung, N. J. \& Na, S. S. The effects of exercise on the GAP-43 expression in the spinal cord of arthritis-induced rats. J. Phys. Ther. Sci., 28(10):2921-3, 2016.

Park, S. J.; Yong, M. S. \& Na, S. S. Effect of exercise on the expression of nerve growth factor in the spinal cord of rats with induced osteoarthritis. J. Phys. Ther: Sci., 27(8):2551-4, 2015.

Pottie, P.; Presle, N.; Terlain, B.; Netter, P.; Mainard, D. \& Berenbaum, F. Obesity and osteoarthritis: more complex than predicted. Ann. Rheum. Dis., 65(11):1403-5, 2006

Qian, J.; Liang, J.; Wang, Y. \& Wang, H. Effect of passive motion on articular cartilage in rat osteoarthritis. Exp. Ther. Med., 8(2):377-83, 2014.

Siebelt, M.; Groen, H. C.; Koelewijn, S. J.; de Blois, E.; Sandker, M.; Waarsing, J. H.; Müller, C.; van Osch, G. J. V. M.; de Jong, M. \& Weinans, H. Increased Physical Activity Severely Induces Osteoarthritic Changes in Knee Joints With Papain Induced Sulfate-Glycosaminoglycan Depleted Cartilage. Arthritis Res. Ther, 16(1):R32, 2014b.

Siebelt, M.; Waarsing, J. H.; Groen, H. C.; Müller, C.; Koelewijn, S. J.; de Blois, E.; Verhaar, J. A. N.; de Jong, M. \& Weinans, H. Inhibited osteoclastic bone resorption through alendronate treatment in rats reduces severe osteoarthritis progression. Bone, 66:163-70, 2014a.

Srinivas, V.; Bohensky, J. \& Shapiro, I. M. Autophagy: a new phase in the maturation of growth plate chondrocytes is regulated by HIF, mTOR and AMP kinase. Cells Tissues Organs, 189(1-4):88-92, 2009.

Sun, H. B. Mechanical loading, cartilage degradation, and arthritis. Ann. N. Y. Acad. Sci., 1211:37-50, 2010.

Tomazoni, S. S.; Leal-Junior, E. C. P.; Frigo, L.; Pallotta, R. C.; Teixeira, S.; de Almeida, P.; Bjordal, J. M. \& Lopes-Martins, R. Á. B. Isolated and combined effects of photobiomodulation therapy, topical nonsteroidal antiinflammatory drugs, and physical activity in the treatment of osteoarthritis induced by papain. J. Biomed. Opt., 21(10):108001, 2016.

Tomazoni, S. S.; Leal-Junior, E. C. P.; Pallotta, R. C.; Teixeira, S.; de Almeida, P. \& Lopes-Martins, R. Á. B. Effects of photobiomodulation therapy, pharmacological therapy, and physical exercise as single and/or combined treatment on the inflammatory response induced by experimental osteoarthritis. Lasers Med. Sci., 32(1):101-8, 2017.

Wenger, H. A. \& Bell, G. J. The interactions of intensity, frequency and duration of exercise training in altering cardiorespiratory fitness. Sports Med., 3(5):346-56, 1986.

Wieland, H. A.; Michaelis, M.; Kirschbaum, B. J. \& Rudolphi, K. A. Osteoarthritis - An untreatable disease? Nat. Rev. Drug Discov., 4(4):331-44, 2005.

Yamaguchi, S.; Aoyama, T.; Ito, A.; Nagai, M.; Iijima, H.; Zhang, X.; Tajino, J. \& Kuroki, H. Effects of exercise level on biomarkers in a rat knee model of osteoarthritis. J. Orthop. Res., 31(7):1026-31, 2013.

Yang, Y.; Wang, Y.; Kong, Y.; Zhang, X.; Zhang, H.; Gang, Y. \& Bai, L. Mechanical stress protects against osteoarthritis via regulation of the AMPK/ NF-kB signaling pathway. J. Cell. Physiol., 234(6):9156-67, 2019.

Zhang, X.; Yang, Y.; Li, X.; Zhang, H.; Gang, Y. \& Bai, L. Alterations of autophagy in knee cartilage by treatment with treadmill exercise in a rat osteoarthritis model. Int. J. Mol. Med., 43(1):336-44, 2019.

Zhou, X.; Wang, W.; Miao, J. \& Bai, L. Expression and significance of transient receptor potential cation channel V5 in articular cartilage cells under exerciseloads. Biomed. Rep., 2(6):813-7, 2014.

Dirección para correspondencia:

Prof. Dr. Nicolás Ernesto Ottone

Laboratorio de Plastinación y Técnicas Anatómcias

Centro de Investigación en Ciencias Odontológicas (CICO)

Facultad de Odontología

Universidad de La Frontera

Av. Francisco Salazar 01145

Casilla 54-D

Temuco

CHILE

Email: nicolas.ottone@ufrontera.cl Recibido : 12-04-2019 Aceptado: 26-06-2019 